\title{
Einige Bemerkungen zu L. VérTES: Das Moustérien in Ungarn
}

\author{
Von Maria Mottl, Graz
}

Im Band 10, 1959, der vorliegenden Zeitschrift ist von L. VÉRTES, Budapest, die obengenannte Zusammenfassung erschienen. Da L. VÉRTEs dabei einige Irrtümer unterlaufen sind, sei es der Verfasserin gestattet, zu diesen rein sachlich Stellung nehmen zu dürfen.

Auf Seite 22 befaßt sich L. VÉRTES mit der zeitlichen Einstufung der Ablagerungen der Subalyuk-Höhle im nördlichen Ungarn, wobei er die Schichten 1-6 in das R/W-Interglazial, die Schichten 7-14 in das W 1 verlegt, wie ich das in der Monographie über die Subalyuk-Höhle schon vor mehr als 20 Jahren getan habe mit dem Unterschied, daß ich eigentlich nur die unteren rotgefärbten Sedimente (Schichten 1-3) als bezeichnend interglaziale untere Schichtgruppe zusammenfaßte, da die Schichten 4 und 6 fundleer waren. L. VÉRTES trifft feinere Unterschiede, indem er einen einleitenden ozeanischen (Schichten 7-9) und einen kontinentalen Abschnitt (Schichten 10-14) des W 1 auseinandertrennt.

L. VÉrtes führt an, daß ich die Schichten 8-17 einheitlich als W 1 bezeichnet hätte, was nicht ganz zutrifft. Zu dieser Angabe möchte ich folgendes bemerken: Ich unterschied wohl eine untere rotgefärbte (Schichten 1-3) und eine obere (grünlichgrau bis hellbraune Schichten, Nr. 7-14 bzw. 17) Schichtgruppe, befaßte mich jedoch, was letztere Schichtgruppe betrifft, hauptsächlich mit der Fauna der hellbraunen Spätmoustérien-Schicht (Schicht 11 und 14), die ich als eine reichhaltige und bezeichnende Tiergemeinschaft dem ausgehenden R/W bzw. dem beginnenden W 1 zugereiht habe. Die Schichten 16-17 (dunkelgrauer, kalkschuttführender und kalkhaltiger schuttführender Lehm mit dem GuloSchädel), die nur im Kamin vorhanden waren, habe ich im Rahmen meiner Faunabearbeitung nicht näher erörtert, wohl aber auf Seite 57 des deutschsprachigen Teiles der Subalyuk-Monographie (Geol. Hungar. Ser. Palaeont. 14, 1939, Budapest) über den GuloFund ausdrücklich gesagt: „Das Exemplar der Subalyuk-Höhle kam aus der dem hellbraunen Höhlenlehm aufgelagerten oberen kalkigen, lößartigen Kaminausfüllung zum Vorschein und gehört wahrscheinlich schon nicht mehr dem Moustérien, sondern einem jüngeren Niveau an.“

Auf Grund seines eigenen Profilrekonstruktionsversuches stellt L. VÉRTES die Schichten 15-16 in das W 1/2 Interstadial und die Schicht 17 mit dem Gulo-Rest (L. VÉrtes gibt nur einen Phalangenknochen an) in das W 2. Wie schwankend diese seine neue Einteilung ist, beweisen seine Sätze auf Seite 66 seines neuerschienenen zusammenfassenden Berichtes: Untersuchungen an Höhlensedimenten (Regeszeti Füzetek 1959, Budapest), indem er schreibt: „Über Schicht 15, die KaDIĆ einen dunkelgrauen, lockeren, kalkschutthaltigen Lehm und MоттL einen dunkelbraunen Lehm nennt, wissen wir kaum etwas. Ihrer Farbe zufolge ist es nicht ausgeschlossen, daß sie das W 1/2 repräsentiert; das ist umso wahrscheinlicher, als die oberste pleistozäne Schicht, 17, annehmbarerweise wieder in einem ozeanischen Abschnitt (W 2 ?) entstand." Auf Seite 124 derselben Publikation wurden Schicht 15 und 17 noch vorsichtiger behandelt und beide, nur mit einem Fragezeichen versehen, diesen Abschnitten zugereiht.

Es soll an dieser Stelle nur mit knappen Sätzen auf einige Mißverständnisse hingewiesen werden, die L. VÉRTES bei seinem Rekonstruktionsversuch unterlaufen sind. Erstens ist die Schicht, die von KaDić im Gesamtprofil der Subalyuk-Höhle — das auf Seite 65 seiner Budapester Publikation auch VÉrTEs bringt - als „dunkelgrauer Höhlenlehm mit lockerem Kalkschutt" bezeichnet wurde, nicht Schicht 15 (das ist nämlich der hellbraune Höhlenlehm mit lockerem Kalkschutt), sondern Schicht 16, und sie ist auch in 
meiner Faunenliste dunkelgrau. Die Schicht wieder, die ich in meiner Faunenliste der Subalyuk-Monographie als dunkelbraunen Lehm anführe, ist Schicht 5 (!) und auch im Profil von KaDIĆ so bezeichnet. Ich schrieb im deutschsprachigen Teil der SubalyukMonographie gleich auf der ersten Seite meiner Faunabearbeitung wörtlich und ausdrücklich: „Die untere Schichtserie gliedert sich petrographisch in eine lebhaftrote plastische (1), eine lebhaftrote Knochenbreccien- (2) und in eine gelblichrote paläolithische Schicht (3). In der oberen Schichtgruppe, welche das Spätmoustérien barg und die sich aus den vom grünlichgrauen (Schicht 7) bis zum hellbraunen Höhlenlehm (14) bzw. bis zum Humus aufeinandergelagerten Schichten zusammensetzt, ist der Höhlenbär die herrschende Gestalt ... Zwischen beiden Schichtgruppen lagerte sich eine dünne Schichtserie $a b$, von deren Schichten die grünlichgelbe (4) und die rotbraune (6) vollkommen steril waren, während die dunkelbraune (5) einige Bärenknochen und Begleitelemente lieferte. Diese Schicht können wir als eine paläontologisch ziemlich scharfe Grenze betrachten, unter und ober welcher uns zwei Säugetiergesellschaften von verschiedener Bedeutung entgegentreten."

In meiner detaillierten Faunenliste der Subalyuk-Monographie sind die Skelettreste einer jeden Art, mit Schichtbezeichnung versehen, in vertikaler Reihenfolge von den oberen bis zu den unteren Schichten zusammengefaßt, wobei innerhalb der oberen Schichtgruppe der dunkelbraune Lehm als untere Grenzschicht gegen die untere Schichtgruppe angeführt wird.

Die Säugetierreste daher, die Vértes auf Grund seiner „Umgruppierung“ auf Seite 23 seines deutschen Berichtes und auf Seite 68 seiner Budapester Publikation als für das W 1/2 der Subalyuk-Höhle ins Feld führt (Sus, Canis, Vulpes, Hyäne, Pferd), haben demzufolge aber schon gar nichts mit Schicht 15 oder $16 \mathrm{zu}$ tun, sondern sie stammen aus Schicht 5, die doch nach VÉrtes (Seite 22 und Seite 66 seiner Publikationen) nach R/W-Interglazial ist!

Auf Seite 29 seiner Studie in Eiszeitalter und Gegenwart behandelt VÉRTES die Sedimente der Ballavölgyer-Höhlung. Er schreibt: „M. MoтtL grub hier 1937-38. Die Ergebnisse der Ausgrabung wurden eigentlich nicht publiziert und außer dem Ausgrabungsprotokoll MotTLs befaßt sich nur ein kurzer Hinweis KaDIĆs mit ihnen." Dann befaßt sich VÉntes mit der Schichtfolge der Höhle, datiert den hellen (gelben) Lehm, meine damalige Auffassung (Spätglazial) revidierend, für Ende R/W oder W 1. In seiner Budapester Publikation entschied er sich auf Seite 126 für den kontinentalen Abschnitt des W 1 und für Anfang W 1/2.

Dazu sei mir erlaubt, folgendes zu berichten: In der Ballavölgyer Höhlung grub ich nicht nur im Jahre 1937, sondern auch im Jahre 1939, und die Ergebnisse wurden von mir, zusammen mit den anderen Ergebnissen meiner vielseitigen Grabungen in den Jahren 1939-40 (Ballahöhle, Tarköer-Felsnische, Háromkuter-Höhle, Gombaszög, DomicaHöhle, Aggteleker-Höhle, Pelsőcardőer-Höhle, Ludmilla-Höhle, Lößforschungen im Gebiete von Nagymaros, Nógrádveröcze, Szob, Basaharc, Pilismarót) in einem ausführlichen Bericht von 37 Seiten mit 39 Abbildungen und zahlreichen Profilzeichnungen niedergeschrieben, wovon 3 Seiten und 5 Abbildungen sich auf die Ballavölgyer-Höhlung bezogen haben. Diese Zusammenfassung für die Jahresberichte der Ung. Geol. Anstalt war im Herbst 1944, zusammen mit dem Bericht über meine Ausgrabungen in den Jahren 1941-42 in Siebenbürgen, sowie mit meinem vierteiligen paläontologischen Bericht: Neue Säugetierfunde in der Sammlung der Ung. Geol. Anstalt zu Budapest (SireniaFunde von Üröm b. Budapest, Die Hipparionfauna von Rudabánya. Anancus arvernensis von Somogyvár und Palaeoloxodon antiquus von Budapest-Várhegy) im Druck, der Umbruch und die Klischeeabzüge von mir bereits korrigiert. Ein Durchschlagexemplar des

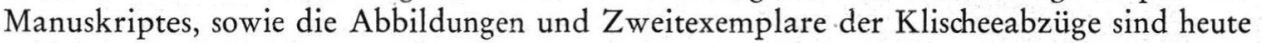
noch in meinem Besitz. 
Da sich, laut VÉRTEs, in der Abteilung für archäologische Dokumentation des Ung. Nationalmuseums von diesen umfangreichen Berichten keine Spur findet, sollen die auf die Ballavölgyer-Höhlung bezogenen Feststellungen hier an Hand des Durchschlagexemplares meines damaligen Berichtes wiedergegeben werden: „Ab Mitte Juli 1939 begann ich mit der weiteren Erschließung der Ballavölgyer Höhlung, in der ich einen Teil der Ausfüllung schon 1937 abtragen ließ. Damals kamen aus der gelblichbraunen Schichte bloß zwei nicht typische, aus Obsidian angefertigte Steingeräte ans Tageslicht, auf Grund welcher kein näherer Kulturhorizont festzustellen war. Die nahezu $8 \mathrm{~m}$ lange, nach untenbergwärts stark ausgedehnte, schön korrodierte Höhlung befindet sich in der Gemarkung der Ortschaft Répáshuta (Komitat Borsod), $10 \mathrm{~m}$ über der Talsohle an der NW-Seite des Kövesváradberges. Die Höhlung entstand entlang NO-SW streichender Spalten zwischen Kalksteinbänken, die unter $45^{\circ}$ einfallen. Die Höhlenausfüllung wies folgende Schichtung auf: 1. Schwarzer Humus. Tierreste: Chiroptera sp. div., Sciurus vulgaris L., Arvicola terrestris L., Microtus arvalis PALL., Cricetus cricetus L., Lepus europaeus PALL., Canis lupus L., Vulpes vulpes L., Meles meles L., Felis silvestris Schreb., Felis catus L., Capreolus capreolus L., Cervus elaphus L., Sus domesticus Gray. - 2. Graubrauner Humus mit vielen neolithischen Topfscherben, einer schönen Neolithperle und den Knochenresten folgender Säugetiere: Erinaceus sp., Ursus arctos L., Vulpes vulpes L., Martes martes L., Cricetus cricetus L., Lepus sp., Capreolus capreolus L., Cervus elaphus L., Sus sp.

Die Tierreste aus dem Humus weisen darauf hin, daß beide Ablagerungen aus jenen Zeiten stammen, als im Bükkgebirge noch Braunbär und Wolf hausten.

3. Gelblichbrauner Höhlenlehm. Eine nahezu $2 \mathrm{~m}$ mächtige Ablagerung, die der Tiefe zu immer grünlicher wird, auch viel Kalkschutt führt, der eckig und autochthonen Ursprungs ist. Die Fauna dieser Schicht ist folgende: Ursus spelaeus Rosenm., Canis lupus L., Vulpes vulpes crucigera BEchst., Alopex lagopus L., Martes martes L., Meles meles L., Mustela erminea L., Hyaena spelaea GoldF., Cricetus cricetus L., Colobotis rufescens K. Blas., Lepus sp., Ochotona pusillus PALL., Capra ibex-Gruppe, Megaceros giganteus Blmb., Rangifer tarandus L., Cervus elaphus L., Rupicapra rupicapra L., Bison priscus BoJ., Lagopus albus K. Br.As., Lagopus mutus Mont., Tetrao tetrix L., Tetrao urogallus L., Tinnunculus tinnunculus L., Asio accipitrinus L.

Höhlenbär und Rentier überwiegen. Auch die Gemse ist häufig, während die anderen Raubtiere und die Nager nur eine untergeordnete Rolle spielen. Die Fuchsreste sind alle kleiner als die unserer typisch hochglazialen Unterart: Vulpes vulpes vulpes L., während sie mit denen der rezenten Vulpes vulpes crucigera BECHST. gut übereinstimmen. Demgegenüber erweisen sich die Zieselreste kräftiger als die des gemeinen Ziesels und entsprechen somit der größeren Art: Colobotis rufescens. Der Rotziesel ist in Ungarn bisher nur aus jüngstpleistozänen Ablagerungen bekannt. Von den Vogelarten deuten die Schneehühner und die Sumpfohreule auf ein kaltes Klima hin, während Birkhuhn, Auerhuhn und der Rüttelfalk Bewohner der gemäßigten Zonen sind.

4. Aus dem unteren, grünlichbraunen Höhlenlehm kamen überwiegend Höhlenbärenreste und nur vereinzelt Wolf-, Hyänen- und Gemsenknochen zutage. D i es er T e il der Höhlenausfüllung ist $z$ weifelsohne älter, während wir den darüberliegenden gelblichbraunen Lehm auf Grund der zahlreichen Rentierknochen und der Anwesenheit von Schneehühnern, Eisfuchs und Sumpfohreule für jünger und zwar für spätglazial halten müssen.

Aus der gelblichbraunen Schichte kamen 1939 mehrere Stein- und Knochenwerkzeuge zum Vorschein. Alle diese Steingeräte, mit Ausnahme einer einzigen Klinge, wurden aus Obsidian angefertigt. Es handelt sich zumeist um dicke, grob zugerichtete, großenteils mit steiler Randretusche bearbeitete Klingenschaber von gedrungener Form. Zwei dünne Klingen stellen typische Geräte des Magdalénien dar. Die eine ist aus Obsidian, schlank, schmal, mit fein gezahnten Rändern, terminal mit einer gut retuschierten Kerbe versehen, 
wodurch das Gerät in einer feinen abgerundeten Bohrerspitze endet. Das Material der anderen, ebenfalls schlanken Klinge ist blauweißer Chalzedonopal. Das langschmale Werkzeug ist nur grob zugerichtet, ohne feinere Retuschierung, und sein oberes Ende ist stichelförmig abgeschrägt. Die Handmanufaktur der Ballavölgyer-Höhlung kann trotz Überwiegen der Klingenschaber nicht dem Aurignacien zugereiht werden. Erstens enthält sie keine typischen Aurignacienformen, zweitens sind ganz ähnliche Stücke auch im heimischen Magdalénien (Kiskevélyer Höhle, Lößstation Ságvár)ํ), aber auch in Magdalénien-Kulturen Deutschlands (Kleine Ofnet, Bocksteinhöhle) und im Schweizersbild anzutreffen. Diese typologische Feststellung wird durch die geborgenen Knochengeräte nur bekräftigt: Ein wunderschön geformtes, fein poliertes, scharfrandiges Gerät stimmt teils mit den entwickelten Pointe-à-cran-Typen, teils mit den triangulären Messerchen des deutschen Spätmagdalénien überein. Auch mehrere, verschieden stark abgenutzte Kiskevélyer Zahnklingen wurden geborgen.

Die auf Grund der Faunazusammensetzung gewonnenen chronologischen Feststellungen werden also durch die aus der Typologie der zum Vorschein gekommenen Stein- und Knochengeräte gezogenen Folgerungen nur unterstützt. "

Soweit mein Berichtsteil über die Ballavölgyer Höhlung, in dem von 20 Stück von mir gesammelten Holzkohlenresten, wie das Vértes in seiner Publikation auf Seite 29 anführt, keine Erwähnung steht, wo doch solche von mir stets immer angegeben worden sind auch dann, wenn ihre endgültige Bestimmung noch ausstand und ihre Untersuchung seitens Univ.-Doz. S. SÁrkánY noch im Gange war.

Ich hoffe damit auch die Sache der Ballavölgyer Höhlung in ein dem Fundmaterial entsprechendes Licht gestellt zu haben.

Manuskr. eingeg. 10. 3. 1960.

Anschr. d. Verfasserin: Kustos Dr. Maria Mottl, Museum Joanneum, Rauberg 10, Graz, Ósterreich.

1) Heute vielfach Höhlengravettien genannt. 